\title{
Test of Special Relativity and Equivalence Principle from Neutrinoless Double Beta Decay
}

\author{
H.V. Klapdor-Kleingrothaus ${ }^{1}$, H. Päs ${ }^{1}$, U. Sarkar ${ }^{2,3}$ \\ ${ }^{1}$ Max-Planck-Institut für Kernphysik P.O. Box 103980, D-69029 Heidelberg, Germany \\ 2 DESY, Notkestrasse 85, D-22607 Hamburg, Germany \\ 3 Physical Research Laboratory, Ahmedabad, 380 009, India
}

(August 7, 2021)

We generalize the formalism for testing Lorentz invariance and the equivalence principle in the neutrino sector. While neutrino oscillation bounds constrain the region of large mixing of the the weak and gravitational eigenstates, we obtain new constraints on violations of Lorentz invariance and the equivalence principle from neutrinoless double beta decay. These bounds apply even in the case of no mixing and thus probe a totally unconstrained region in the parameter space.

Special relativity and the equivalence principle can be considered as the most basic foundations of the theory of gravity. Many experiments already have tested these principles to a very high level of accuracy [1] for ordinary matter - generally for quarks and leptons of the first generation. These precision tests of local Lorentz invariance - violation of the equivalence principle should produce a similar effect 2] - probe for any dependence of the (non-gravitational) laws of physics on a laboratory's position, orientation or velocity relative to some preferred frame of reference, such as the frame in which the cosmic microwave background is isotropic.

A typical feature of the violation of local Lorentz invariance (VLI) is that different species of matter have a characteristical maximum attainable speed. This can be tested in various sectors of the standard model through vacuum Cerenkov radiation [3], photon decay [4, neutrino oscillations [5,8, 11] and $K$-physics [6,7]. In this article we extend these arguments to derive new constraints from neutrinoless double beta decay.

The equivalence principle implies that spacetime is described by unique operational geometry and hence universality of the gravitational coupling for all species of matter. In the recent years there have been attempts to constrain a possible amount of violation of the equivalence principle (VEP) in the neutrino sector from neutrino oscillation experiments 8 11. However, these bounds don't apply when the gravitational and the weak eigenstates have small mixing. In this article we present a generalized formalism of the neutrino sector to test the VEP and point out that neutrinoless double beta decay also constrains the VEP. VEP implies different neutrino species to suffer from different gravitational potentials while propagating through the nucleus and hence the effect of different eigenvalues doesn't cancel for the same effective momentum. Earlier results on neutrino oscillations come out as special case from our present formalism. The main result is that neutrinoless double beta decay can constrain the amount of VEP even when the mixing angle is zero, i.e., when only the weak equivalence principle is violated, for which there does not exist any bound at present.

We shall first present our formalism for VLI and then for VEP. For sake of clarity we formulate the problem for a two generation scenario involving $\nu_{e}$ and $\nu_{x}$ with $x=\mu, \tau, s$. Neutrinos of different species may have different maximum attainable velocities if there is violation of local Lorentz invariance (VLI) and hence violation of special relativity [1]. We first assume that the weak eigenstates cannot be diagonalized simultaneously with the velocity eigenstates and the neutrinos are relativistic point particles. The effective Hamiltonian in the weak basis $\left[\begin{array}{ll}\nu_{e} & \nu_{x}\end{array}\right]$ is

$$
H=U_{m} H_{m} U_{m}^{-1}+U_{v} H_{v} U_{v}^{-1} .
$$

In absence of VLI the neutrino mass matrix in the mass basis $\left[\begin{array}{ll}\nu_{1} & \nu_{2}\end{array}\right]$ is given by

$$
H_{m}=\frac{\left(M_{m}\right)^{2}}{2 p}=\frac{1}{2 p}\left(\begin{array}{cc}
m_{1} & 0 \\
0 & m_{2}
\end{array}\right)^{2}
$$

and the VLI part of the hamiltonian as

$$
H_{v}=\left(\begin{array}{cc}
v_{1} & 0 \\
0 & v_{2}
\end{array}\right) p
$$

to leading order in $\bar{m}^{2} / p^{2}$. Here $\mathrm{p}$ denotes the momentum and $\bar{m}$ the average mass, and for any quantity $X$ we define $\delta X \equiv\left(X_{1}-X_{2}\right), \bar{X}=\left(X_{1}+X_{2}\right) / 2$.

In the absence of VLI, i.e., when the special threory of relativity is valid, $v_{i}=1$, and $H_{v}$ simply becomes the momentum of the neutrinos. Here we are interested in a single neutrino beam (for neutrino oscillation experiments) or a single virtual neutrino propagating inside the nucleus with a particular momentum. For this reason we assume the momenta of both the neutrinos are $p$. Then $v_{i}$ corresponds to the maximum attainable speed of the corresponding momentum eigenstates. Hence $v_{1}-v_{2}=\delta v$ is a measure of VLI in the neutrino sector. As typical or "standard" maximum attainable speed $\frac{v_{1}+v_{2}}{2}=1$ is assumed. All previous bounds on this quantity $\delta v$ in the neutrino sector were derived from neutrino oscillation experiments and for that reason these bounds are valid only 
for large gravitational mixing. As we shall point out, neutrinoless double beta decay can constrain $\delta v$ even when the mixing angle vanishes.

We shall not consider any $C P$ violation, and hence $H_{m}$ and $H_{v}$ are real symmetric matrices and $U_{m}$ and $U_{v}$ are orthogonal matrices $U^{-1}=U^{T}$. They can be parametrized as $U_{i}=\left(\begin{array}{cc}\cos \theta_{i} & \sin \theta_{i} \\ -\sin \theta_{i} & \cos \theta_{i}\end{array}\right)$, where $\theta_{i}$ represents weak mixing angle $\theta_{m}$ or velocity mixing angle $\theta_{v}$. We can now write down the weak Hamiltonian $H_{w}$ in the basis $\left[\begin{array}{ll}\nu_{e} & \nu_{x}\end{array}\right]$, in which the charged lepton mass matrix is diagonal and the charged current interaction is also diagonal, as

$$
H=p I+\frac{1}{2 p}\left(\begin{array}{cc}
M_{+} & M_{12} \\
M_{12} & M_{-}
\end{array}\right)^{2} .
$$

Here $I$ is the identity matrix and

$$
\begin{aligned}
M_{ \pm}= & \bar{m} \pm \frac{\cos 2 \theta_{m}}{2} \delta m \\
& \pm \frac{p^{2}}{\bar{m}} \delta v\left(\frac{\cos 2 \theta_{v}}{2}-\frac{\delta m}{4 \bar{m}} \cos 2\left(\theta_{m}-\theta_{v}\right)\right) \\
M_{12}= & -\frac{\sin 2 \theta_{m}}{2} \delta m \\
& -\frac{p^{2}}{\bar{m}} \delta v\left(\frac{\sin 2 \theta_{v}}{2}+\frac{\delta m}{4 \bar{m}} \sin 2\left(\theta_{m}-\theta_{v}\right)\right) .
\end{aligned}
$$

In the case of exact Lorentz invariance, we usually write the mass matrix in the weak basis as $\left(\begin{array}{cc}m_{e e} & m_{e \mu} \\ m_{e \mu} & m_{\mu \mu}\end{array}\right)$. In the mass mechanism of neutrinoless double beta decay, the decay rate

$$
\left[T_{1 / 2}^{0 \nu \beta \beta}\right]^{-1}=\frac{\langle m\rangle^{2}}{m_{e}^{2}} G_{01}|M E|^{2}
$$

is proportional to the effective neutrino mass $\langle m\rangle=$ $m_{e e}=M_{+}$. Here $M E$ denotes the nuclear matrix element $M E=M_{F}-M_{G T}, G_{01}$ corresponds to the phase space factor defined in [12] and $m_{e}$ is the electron mass. The double beta observable can be written as

$$
\begin{aligned}
<m> & =\sum_{i} U_{e i}^{2} m_{i}=m_{1} \cos ^{2} \theta_{w}+m_{2} \sin ^{2} \theta_{w} \\
& =\bar{m}+\frac{1}{2} \delta m \cos 2 \theta_{w} .
\end{aligned}
$$

If $m_{e e}=0$, the two physical eigenstates with eigenvalues $m_{1}$ and $m_{2}$ will contribute to the neutrinoless double beta decay by an amount $U_{e 1}^{2} m_{1}$ and $U_{e 2}^{2} m_{2}$, respectively, which cancels each other. However, if these two physical states have different maximum attainable speed, corresponding to VLI, this cancellation will not be exact for the same cut-off effective momentum in the neutrino propagator. As a result, even when $m_{e e}=0$, we can have neutrinoless double beta decay, which is proportional to the amount of VLI and the double beta observable is given by $M_{+}$in (舟). From (舟) it can easily be seen that in the region of maximal mixing $\cos 2 \theta_{v}=0$, the double beta decay rate vanishes. Thus neutrinoless double beta decay doesn't constrain the amount of VLI for maximal mixing. However, when the mixing approaches zero, the most stringent bound from neutrinoless double beta decay is obtained. In this case $\delta v / 2$ can be understood as derivation from the standard maximum attainable speed $\bar{v}$. As it is obvious, when there is no mixing the neutrino oscillation experiments cannot give any bound on the amount of VLI, since in absence of mixing only VLI cannot allow neutrino oscillations.

To give a bound on VLI in the small mixing region (including $\theta_{v}=\theta_{m}=0$ ) we assume conservatively $\langle m\rangle \simeq 0$. We also assume $\delta m \leq \bar{m}$, and thus $\frac{\delta m}{4 \bar{m}}$ may be neglected. Due to the $p^{2}$ enhancement the nuclear matrix elements of the mass mechanism have to be replaced by $\frac{m_{p}}{R} \cdot\left(M_{F}^{\prime}-M_{G T}^{\prime}\right)$ with the nuclear radius $R$ and the proton mass $m_{p}$, which have been calculated in [13. Inserting the recent half life limit obtained from the Heidelberg-Moscow experiment [14], $T_{1 / 2}^{0 \nu \beta \beta}>1.2 \cdot 10^{25} y$, a bound on the amount of VLI as a function of the average neutrino mass $\bar{m}$ can be given. The most reliable assumption for $\bar{m}$ is obtained from the cosmological bound $\sum_{i} m_{i}<40 \mathrm{eV} 15$, i.e., $\bar{m}<13 \mathrm{eV}$ for three generations, implying a bound of

$$
\delta v<4 \times 10^{-16} \text { for } \theta_{v}=\theta_{m}=0 .
$$

However, combining the present experimental constraints from atmospheric and solar neutrino data as well as from tritium beta decay in a three neutrino framework and assuming a typical hierarchical mass pattern spectrum $m_{3} \gg m_{1,2}$ or $m_{3} \simeq m_{2} \gg m_{1}$ implies $\bar{m}<\sim 0.08$ $\mathrm{eV}$ [16 and improves the bound to $\delta v<2 \cdot 10^{-18}$ for $\theta_{v}=\theta_{m}=0$.

In figure 1 the bound implied by double beta decay is presented for the entire range of $\sin ^{2} 2 \theta_{v}$ and compared with bounds obtained from neutrino oscillation experiments in [10]. It should be stressed also that the GENIUS proposal of the Heidelberg group [17] could improve these bounds by about 1-2 orders of magnitude.

For comparison, in the following the amount of VLI in neutrino oscillation experiments is calculated in this formalism. In the basis of the physical states $\nu_{a}$ and $\nu_{b}$, the Hamiltonian becomes

$$
\begin{aligned}
H & =\left(\begin{array}{cc}
p+\frac{m_{a}^{2}}{2 p} & 0 \\
0 & p+\frac{m_{b}^{2}}{2 p}
\end{array}\right) \\
& =\left(\begin{array}{cc}
\bar{E} & 0 \\
0 & \bar{E}
\end{array}\right)+\frac{1}{2}\left(\begin{array}{cc}
\Delta E & 0 \\
0 & -\Delta E
\end{array}\right),
\end{aligned}
$$

where $\bar{E}=\left(p+\frac{\bar{m}^{2}}{2 p}\right)$ and

$$
\begin{aligned}
& \frac{p}{\bar{m}} \Delta E=m_{a}-m_{b} \\
& =\left[(\delta m)^{2}+\left(\delta v \frac{p^{2}}{\bar{m}}\right)^{2}+2 \delta m \delta v \frac{p^{2}}{\bar{m}} \cos \left(2\left(\theta_{w}-\theta_{v}\right)\right)\right]^{1 / 2} .
\end{aligned}
$$


The new mixing angle $\theta_{\text {tot }}$ is a function of $\theta_{m}$ and $\theta_{v}$ and the oscillation probability is now given by

$$
P\left(\nu_{e} \rightarrow \nu_{x}\right)=\sin ^{2} 2 \theta_{t o t} \sin ^{2} \frac{\pi L}{\lambda},
$$

where $\lambda=\frac{\pi p}{\Delta m^{2}}$ and $\Delta m^{2}=m_{a}^{2}-m_{b}^{2}$. In the limit of vanishing neutrino masses, neutrino oscillations are implied only by VLI. In this case the oscillation probability becomes

$$
P\left(\nu_{e} \rightarrow \nu_{x}\right)=\sin ^{2} 2 \theta_{v} \sin ^{2} p L \delta v,
$$

which corresponds to the expression obtained earlier [5]. Here $p$ denotes the total beam energy. From this expression it beomes clear that in the case of no mixing, $\theta_{v}=0$, neutrino oscillation experiments don't constrain the size of VLI effects.

In the following we present the formalism for violation of the equivalence principle (VEP). While in the final expression the amount of VLI just will be replaced by VEP, the origin differs. In a linearized theory the gravitational part of the Lagrangian to first order in a weak gravitational field $g_{\mu \nu}=\eta_{\mu \nu}+h_{\mu \nu}\left(h_{\mu \nu}=2 \frac{\phi}{c^{2}} \operatorname{diag}(1,1,1,1)\right)$ can be written as $\mathcal{L}=-\frac{1}{2}\left(1+g_{i}\right) h_{\mu \nu} T^{\mu \nu}$, where $T^{\mu \nu}$ is the stress-energy in the gravitational eigenbasis. In the presence of VEP the $g_{i}$ may differ. Assuming only violation of the weak equivalence principle, the gravitational interaction is diagonal but the couplings differ. In this case there does not exist any bound on the amount of VEP. We point out that this region of the parameter region is most restrictively bounded by neutrinoless double beta decay.

The effective Hamiltonian in the weak basis again can be written as

$$
H=p I+U_{m} H_{m} U_{m}^{-1}+U_{G} H_{G} U_{G}^{-1},
$$

with $H_{m}$ given in (2) and

$$
\begin{aligned}
H_{G} & =\left(\begin{array}{cc}
G_{1} & 0 \\
0 & G_{2}
\end{array}\right) \\
& =\left(\begin{array}{cc}
-2\left(1+g_{1}\right) \phi\left(p+\frac{\bar{m}^{2}}{2 p}\right) & 0 \\
0 & -2\left(1+g_{2}\right) \phi\left(p+\frac{\bar{m}^{2}}{2 p}\right)
\end{array}\right)
\end{aligned}
$$

to first order in $\bar{m}^{2} / p^{2}$. In formalisms where only violation of the weak equivalence principle is assumed, one starts with $U_{G}$ proportional to $U_{W}$, in which case there does not exist any bound from the neutrino oscillation experiments.

The Hamiltonian in the weak basis is

$$
\begin{aligned}
M_{ \pm}= & \bar{m} \pm \frac{\cos 2 \theta_{m}}{2} \delta m \\
& \pm \frac{p}{\bar{m}} \delta G\left(\frac{\cos 2 \theta_{G}}{2}-\frac{\delta m}{4 \bar{m}} \cos \left(\theta_{m}-\theta_{v}\right)\right) \\
M_{12}= & -\frac{\sin 2 \theta_{m}}{2} \delta m \\
& -\frac{p}{\bar{m}} \delta G\left(\frac{\sin 2 \theta_{G}}{2}+\frac{\delta m}{4 \bar{m}} \sin \left(\theta_{m}-\theta_{v}\right)\right) .
\end{aligned}
$$

Compared to VLI, the expressions for this case of VEP remain unchanged, except for replacing $\delta v$ by $\frac{1}{p} \delta G$. Again, $\bar{g}=\frac{g_{1}+g_{2}}{2}$ can be considered as the standard gravitational coupling, for which the equivalence principle applies.

Thus the discussion of VLI can be directly translated to the VEP case and the bound from neutrinoless double beta decay for $\theta_{v}=\theta_{m}=0$ is now given by

$$
\begin{array}{r}
\phi \delta g<4 \times 10^{-16} \mathrm{GeV}(\text { for } \bar{m}<13 \mathrm{eV}) \\
\phi \delta g<2 \times 10^{-18} \mathrm{GeV}(\text { for } \bar{m}<0.08 \mathrm{eV})
\end{array}
$$

In this case, $\delta G=p \phi \delta g$, where $\phi$ is the background Newtonian gravitational potential on the surface of the earth. A natural choice for $\phi$ would be the earth's gravitational potential $\left(\sim 10^{-9}\right)$, but another well motivated choice could be the potential due to all forms of distant matter. Unlike the case of VLI, the bound on the VEP will depend on what one chooses for the Newtonian potential $\phi$. For this reason, here we only present the combined bound on $\phi \delta g$.

In summary, we presented a general formalism for the study of both VLI and VEP in the neutrino sector. We pointed out that neutrinoless double beta decay can constrain the amount of VLI or VEP. In particular, when the mixing of the gravitational eigenstates vanishes, the bounds from neutrinoless double beta decay become most stringent, while this region is not constrained by any other experiments.

Acknowledgement One of us (US) would like to acknowledge the hospitality of the Theory Group, DESY, Hamburg, the Max-Planck-Institut für Kernphysik, Heidelberg and a fellowship from the Alexander von Humboldt Foundation.

1] V.W. Hughes, H.G. Robinson, and V. Beltran-Lopez, Phys. Rev. Lett. 4, 342 (1960); R.W.P. Drever, Philos. Mag. 6, 683 (1961); D. Newman, G.W. Ford, A. Rich and E. Sweetman, Phys. Rev. Lett. 40, 1355 (1978); A. Brillet and J.L. Hall, Phys. Rev. Lett. 42, 549 (1979); J.D. Prestage, J.J. Bollinger, W.M. Itano, and D.J. Wineland, Phys. Rev. Lett. 54, 2387 (1985); S.K. Lamoureaux, J.P. Jacobs, B.R. Heckel, R.J. Raab, and E.N. Fortson, Phys. Rev. Lett. 57, 3125 (1986).

[2] C. M. Will, Theory and Experiment in Gravitational Physics, 2nd edition (Cambridge University Press, Cambridge, 1992).

[3] M. Gasperini, Phys. Rev. Lett. 62, 1945 (1989).

[4] S. Coleman and S.L. Glashow, Phys. Lett. B 405, 249 (1997).

[5] S.L. Glashow, A. Halprin, P.I. Krastev, C.N. Leung, and J. Panteleone, Phys. Rev. D 56, 2433 (1997).

[6] T. Hambye, R.B. Mann and U. Sarkar, Phys. Lett. B 421, 105 (1998); Phys. Rev. D 58, 025003 (1998). 
[7] M.L. Good, Phys. Rev. 121, 311 (1961); O. Nachtmann, Acta Physica Austriaca, Supp. VI Particle Physics ed. P. Urban, (1969) p. 485; S.H. Aronson, G.J. Bock, HY Cheng and E. Fishbach, 48, 1306 (1982); Phys. Rev. D28, 495 (1983); I.R. Kenyon, Phys. Lett. B237, 274 (1990); R.J. Hughes, Phys. Rev. D46, R2283 (1992);

[8] M. Gasperini, Phys. Rev. D38, 2635 (1988); ibid. D39, 3606 (1989).

[9] A. Halprin and C.N. Leung, Phys. Rev. Lett. 67, 1833 (1991); Nucl. Phys. B28A (Proc. Supp.), 139 (1992); J. N. Bahcall, P. I. Krastev, and C. N. Leung, Phys. Rev. D52, 1770 (1995); R.B. Mann and U. Sarkar, Phys. Rev. Lett 76 (1996) 865.

[10] A. Halprin, C.N. Leung, J. Pantalone, Phys.Rev. D 53 (1996) 5365

[11] M. N. Butler et al., Phys. Rev. D47, 2615 (1993); A. Halprin and C. N. Leung, Phys. Rev. Lett. 67, 1833 (1991); J. Pantaleone, A. Halprin, and C. N. Leung, Phys. Rev. D47, R4199 (1993); K. Iida, H. Minakata and O. Yasuda, Mod. Phys. Lett. A8 (1993) 1037.

[12] M. Doi, T. Kotani, E. Takasugi, Progr. Theor. Phys. Suppl. 83 (1985) 1

[13] H. Päs, M. Hirsch, S.G. Kovalenko, H.V. KlapdorKleingrothaus, subm. to Phys. Rev . Lett. M. Hirsch, H.V. Klapdor-Kleingrothaus, S.G. Kovalenko, Phys. Lett. B 372 (1996) 181, Erratum: Phys. Lett. B 381 (1996) 488

[14] H.V. Klapdor-Kleingrothaus, H. Päs, Proc. of the 6th Int. Symposium on Particles, Strings and Cosmology, (PASCOS98), Boston(MA), USA, March 22-27 1998, to be publ. by World Scientific; M. Günther et al, Phys. Rev. D 55 (1997) 54; L. Baudis et al, Phys. Lett. B 407 (1997) 219.

[15] G. Raffelt in Proc. TAUP97, Nucl. Phys. B (Proc. Suppl.) 70 (1998) 169

[16] V. Barger, T.J. Weiler, K. Whisnant, hep-ph/9808367

[17] H.V. Klapdor-Kleingrothaus, hep-ex/9802007, in: H.V. Klapdor-Kleingrothaus, H. Päs (Eds.), Proc. Int. Conf. "Beyond the Desert - Accelerator- and Non-Accelerator Approaches", Castle Ringberg, Germany, 1997; H.V. Klapdor-Kleingrothaus, M. Hirsch, Z. Phys. A 359 (1997) 361; H.V. Klapdor-Kleingrothaus, J. Hellmig, M. Hirsch, J. Phys. G 24 (1998) 483;

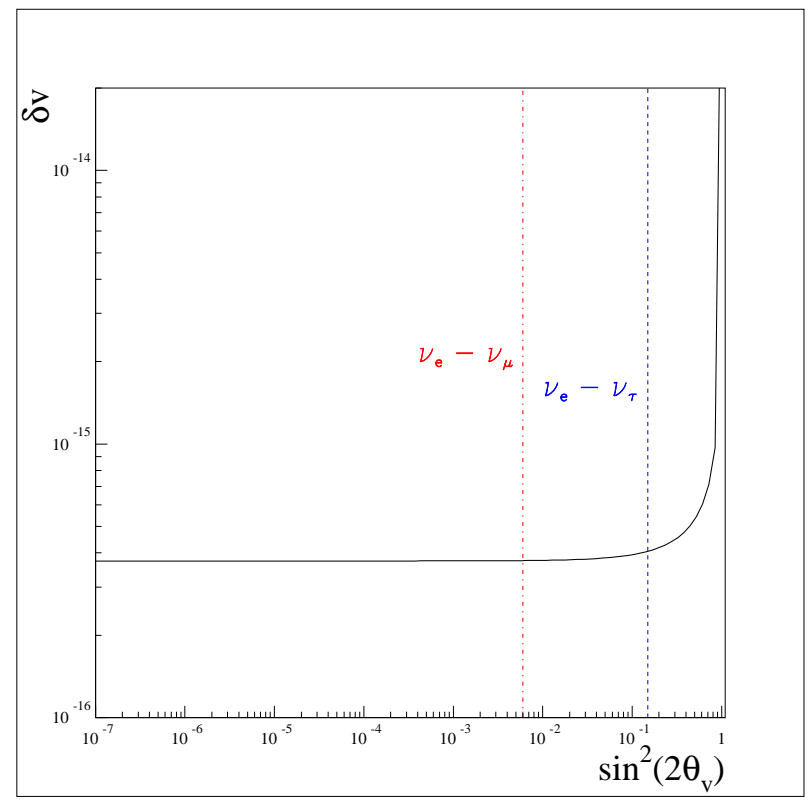

FIG. 1. Double beta decay bound (solid line) on violation of Lorentz invariance in the neutrino sector, excluding the region to the upper left. Shown is a double logarithmic plot in the $\delta v-\sin ^{2}(2 \theta)$ parameter space. The bound becomes most stringent for the small mixing region, which has not been constrained from any other experiments. For comparison the bounds obtained from neutrino oscillation experiments (from [10]) in the $\nu_{e}-\nu_{\tau}$ (dashed lines) and in the $\nu_{e}-\nu_{\mu}$ (dashed-dotted lines) channel, excluding the region to the right, are shown. 